\title{
Functional Liquid-Crystalline Materials Based on Ferrocene
}

\author{
Thierry Chuarda and Robert Deschenaux ${ }^{\star}$
}

\begin{abstract}
This short account demonstrates that ferrocene is a valuable unit for the elaboration of functional liquid-crystalline materials: 1) its three-dimensional structure allowed a clear structure-mesomorphic behavior relationship in the case of mono-, di-, and poly-substituted ferrocene-containing liquid crystals to be established; 2) its derivatization at the 1,3-positions by two different substituents generated structures with planar chirality; and 3) its redox properties could be used to produce redox-active mesomorphic liquid crystals.
\end{abstract}

Keywords: Dendrimers · Ferrocene - Liquid crystals · Metallomesogens · Polymers · Supramolecular chemistry

\section{Introduction}

Thermotropic liquid crystals play a crucial role in everyday life as they have found widespread applications in the manufacture of e.g. watches, calculators, mobile telephones, notebook computers, thermometry, specific oils, and pigments. Further applications are expected in the future. Achievement of this goal requires the design of liquid-crystalline materials with novel properties. The development of functional liquid crystals with tailor-made properties is a current challenge in materials science.

Incorporation of active subunits into liquid crystals should give rise to such new, highly efficient materials, which will combine the properties of the subunits with those of liquid crystals (organization, anisotropy).

\footnotetext{
${ }^{*}$ Correspondence: Prof. R. Deschenaux Institut de Chimie

Université de Neuchâtel

Avenue de Bellevaux 51

Case postale 2

CH-2007 Neuchâtel

Tel.: +41327182400

Fax: +41327182511

E-Mail: robert.deschenaux@unine.ch

aCurrent address: Lehrerinnen- und Lehrerbildung

Universität Bern

Departement für Chemie und Biochemie

Freiestrasse 3

$\mathrm{CH}-3012$ Bern
}

If specific properties are to be exploited (magnetic properties, redox properties, optical properties, catalytic properties), the choice of the subunit and how it is connected to the liquid crystal are of prime importance.

Our interest in supramolecular materials motivated us to develop thermotropic liquid crystals incorporating various subunits. These were selected in view of their structural characteristics and specific properties. The subunits we have incorporated into liquid crystals are:

1) ferrocene (redox-active unit) [1],

2) ruthenocene (redox-active unit) [2],

3) $\left(\eta^{6}\right.$-arene)tricarbonylchromium complexes (photo-active unit) [3],

4) bisruthenium clusters (catalytically-active units) [4], and

5) fullerene (redox- and photo-active unit) [5].

From a fundamental point of view, the incorporation of such subunits into anisotropic materials is interesting in order to establish a structure-supramolecular organization relationship and gain novel information which can be useful for the development of other anisotropic assemblies such as lyotropic liquid crystals, micelles, and membranes.

The scope of this account is to highlight, with selected examples, some relevant results we have obtained in case of ferrocenecontaining thermotropic liquid crystals. For details and in-depth discussion, the readers are referred to the papers and reviews mentioned in the text.

\section{Properties}

\subsection{Influence of the Substitution Pattern on the Liquid-Crystalline Properties}

The three-dimensional structure of ferrocene allowed the synthesis of a great variety of derivatives, such as mono- [6], di[1], and 1,1',3-tri-substituted liquid-crystalline ferrocenes [7]. The substitution pattern has a strong influence on the formation and stability of liquid-crystalline phases. The following mesomorphic tendency was observed: mono-substitution $<$ di-substitution $<1,1$ ',3-tri-substitution. This sequence can be explained in terms of intermolecular interactions which increase with the number of substituents. The disubstitution pattern gives rise to three isomers for which the liquid crystal tendency depends on the position of the substituents (Fig. 1). The following liquid crystal tendency was observed: $1,2-<1,1^{\prime}-<1,3$-isomeric structures. This sequence can be explained in terms of structural features as:

i) the 1,3-disubstituted ferrocenes possess the highest molecular anisotropy among the isomeric structures;

ii) the substituents located at the 1,1 '-positions generate a step in the structure, the consequence of which is a reduction of the molecular anisotropy and a loss of co-linearity of the mesogenic substituents; and

iii) the substituents located in the 1,2-positions generate a hairpin structure which lacks molecular anisotropy. 


\subsection{Liquid-Crystalline Ferrocenes with Planar Chirality}

Unsymmetrically 1,3-disubstituted ferrocenes are interesting materials as they combine structural anisotropy and planar chirality [9]. Such compounds are suitable candidates for the elaboration of ferroelectric liquid crystals for which the properties can be controlled as a function of the mesogenic groups that are grafted onto the organometallic unit. Ferrocene 4 (Fig. 2) was obtained with an enantiomeric excess of $98 \%$ and its spontaneous polarization was determined as $2.8 \mathrm{nC} \cdot \mathrm{cm}^{-2}$. This value is consistent with the structure which carries two organic fragments that are differentiated only by the length of the terminal alkyl chains and the orientation of the external ester functions. As most of the studies on chiral liquid crystals are developed with compounds having point chirality, planar chirality is an alternative and elegant way to obtain optically-active materials.

\subsection{Ferrocene-Containing Side-Chain Liquid-Crystalline Polymers}

Ferrocene-containing liquid-crystalline main-chain polymers were shown to exhibit limited thermal stability making their characterization difficult [10]. We turned our attention to side-chain polymers, i.e. polysiloxanes and polymethacrylates (Fig. 3 ). We anticipated that such polymers could be prepared from vinyl- and methacrylatecontaining ferrocene monomers, respectively, following well-established procedures developed for organic monomers. Polysiloxane 5 [11] was prepared by grafting the corresponding vinyl monomer (structure not shown) onto commercially available polyhydrosiloxane following a standard procedure (toluene, $70{ }^{\circ} \mathrm{C}, 24 \mathrm{~h}$, $\left.\mathrm{PtCl}_{2}\left(1,5-\mathrm{C}_{8} \mathrm{H}_{12}\right)\right)$. Polymethacrylates 6 [12] and 7 [13] were prepared by free-radical polymerization (THF, AIBN, $50{ }^{\circ} \mathrm{C}$ ) of the corresponding methacrylate monomers (structures not shown). Polymers 5-7 showed good solubility in common organic solvents $\left(\mathrm{CH}_{2} \mathrm{Cl}_{2}, \mathrm{CHCl}_{3}\right.$, THF), good thermal stability (no decomposition was detected up to $250{ }^{\circ} \mathrm{C}$ ), and narrow molecular weight distribution (Mw/Mn: 1.4-1.6).

\subsection{Redox-Active \\ Liquid-Crystalline Ferrocenes}

Ferrocene has found interesting applications as an electroactive building block for elaborating switchable molecular aggregates [14], redox-active receptors [15], redox-active polymeric ionomers [16], and conducting and magnetic materials [17]. With the aim of developing electroactive liquid-crystalline materials, we synthesized

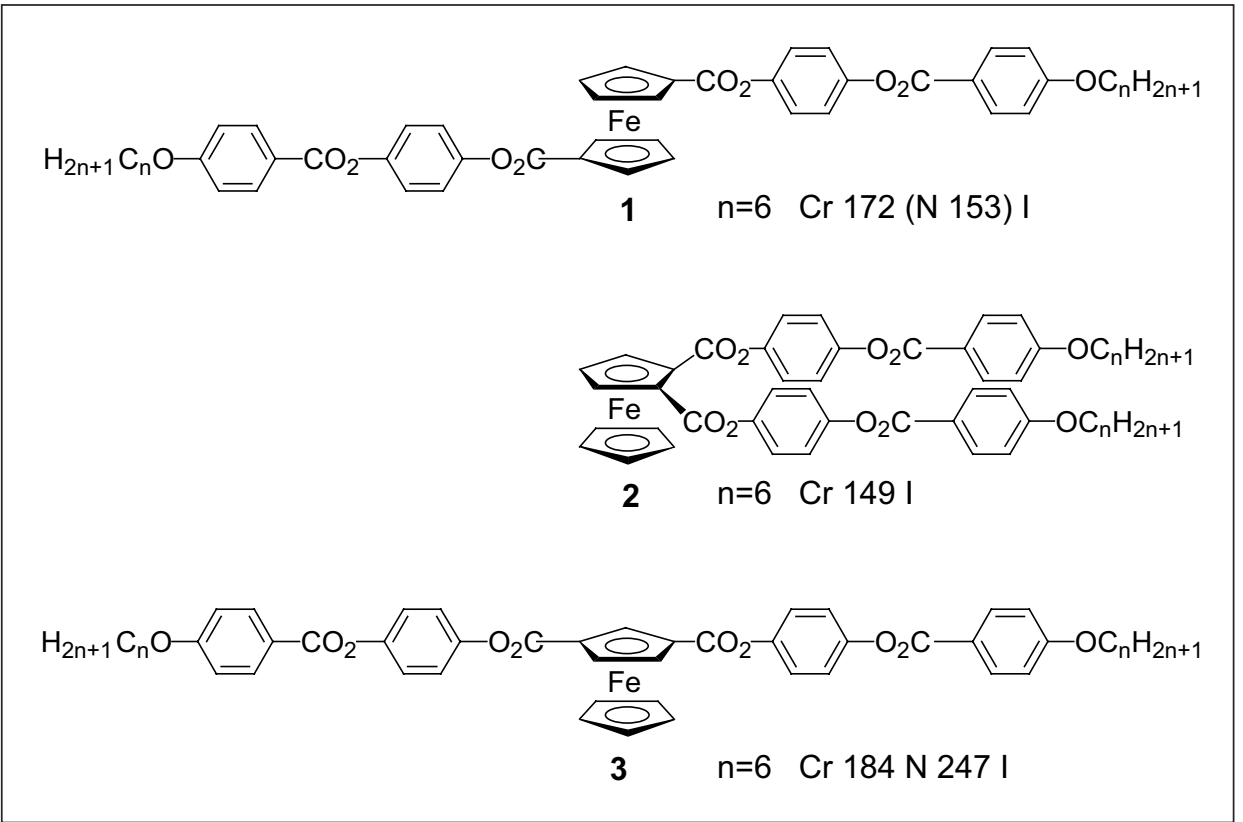

Fig. 1. Isomeric 1,1'- (1), 1,2- (2) and 1,3 (3)-disubstituted ferrocene derivatives and their thermal and liquid-crystalline properties. For abbreviations, see [8].

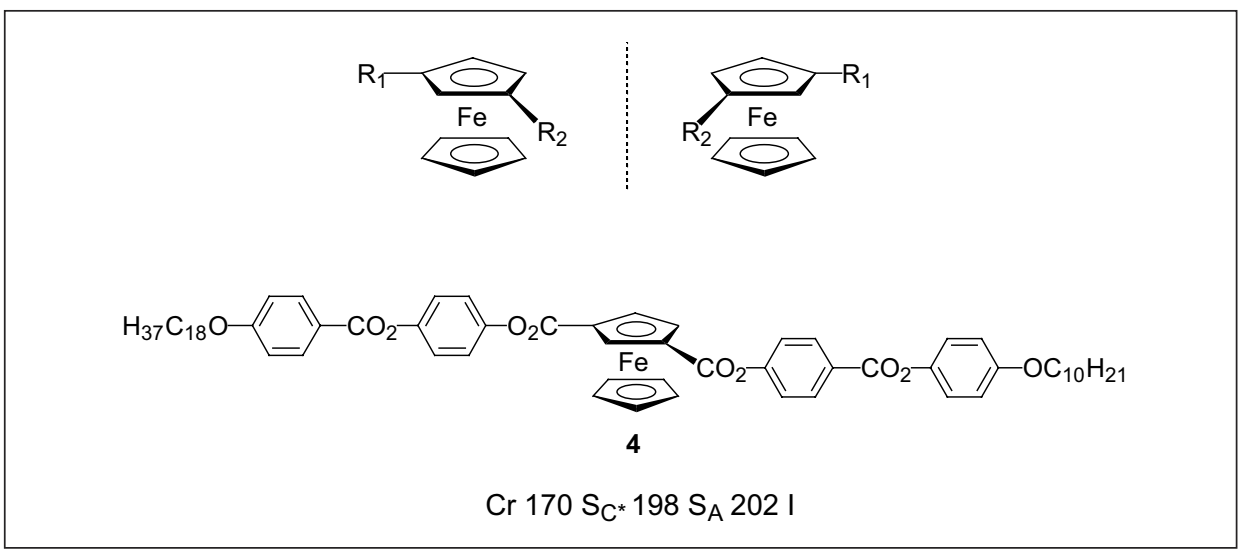

Fig. 2. Planar chirality in unsymmetrically 1,3 -disubstituted ferrocene derivatives, and an optically-active ferrocene-containing liquid crystal. For abbreviations, see [8].

8 and 9 (Fig. 4). We used peralkylated ferrocenes because of the ease of oxidation of such units. Compounds $8(\rightarrow \mathbf{1 0})$ [18] and $9(\rightarrow 11)[19]$ were oxidized with silver tosylate. Polymer $7(\rightarrow \mathbf{1 2})$ was oxidized with iodine (Fig. 4) [20]. Oxidation had a clear influence on the thermal and mesomorphic properties. Indeed, while $\mathbf{8}$ and 9 were not mesomorphic, the corresponding ferrocenium derivatives showed smectic A or columnar phases, respectively, and while polymer 7 exhibited smectic phases, its oxidized form gave rise to the nematic phase.

\subsection{Ferrocene-Containing Liquid-Crystalline Dendrimers}

Dendrimers represent a class of materials which combine unique features (welldefined macromolecular structure, monodispersity, low viscosity) with remarkable properties (encapsulation, catalysis, chiroptical properties) [21]. Functionalized dendrimers, i.e. dendrimers incorporating active or reactive functions, are considered as new materials with potential applications, such as for the preparation of macromolecular libraries [22]. With the view to combine the properties and features of dendrimers with the properties of ferrocene, we designed and synthesized the second-generation liquid-crystalline dendrimer $\mathbf{1 3}$ (Fig. 5) in which cholesterol acts as liquidcrystalline promoter [23]. Dendrimer 13 was analyzed by cyclic voltametry, which revealed a reversible oxidation process with twelve electrons being transferred. A value of $0.93 \mathrm{~V}$ was obtained for the redox potential.

\section{Conclusion}

Ferrocene-containing liquid crystals have reached a high degree of complexity that can be used to elaborate mesomorphic 


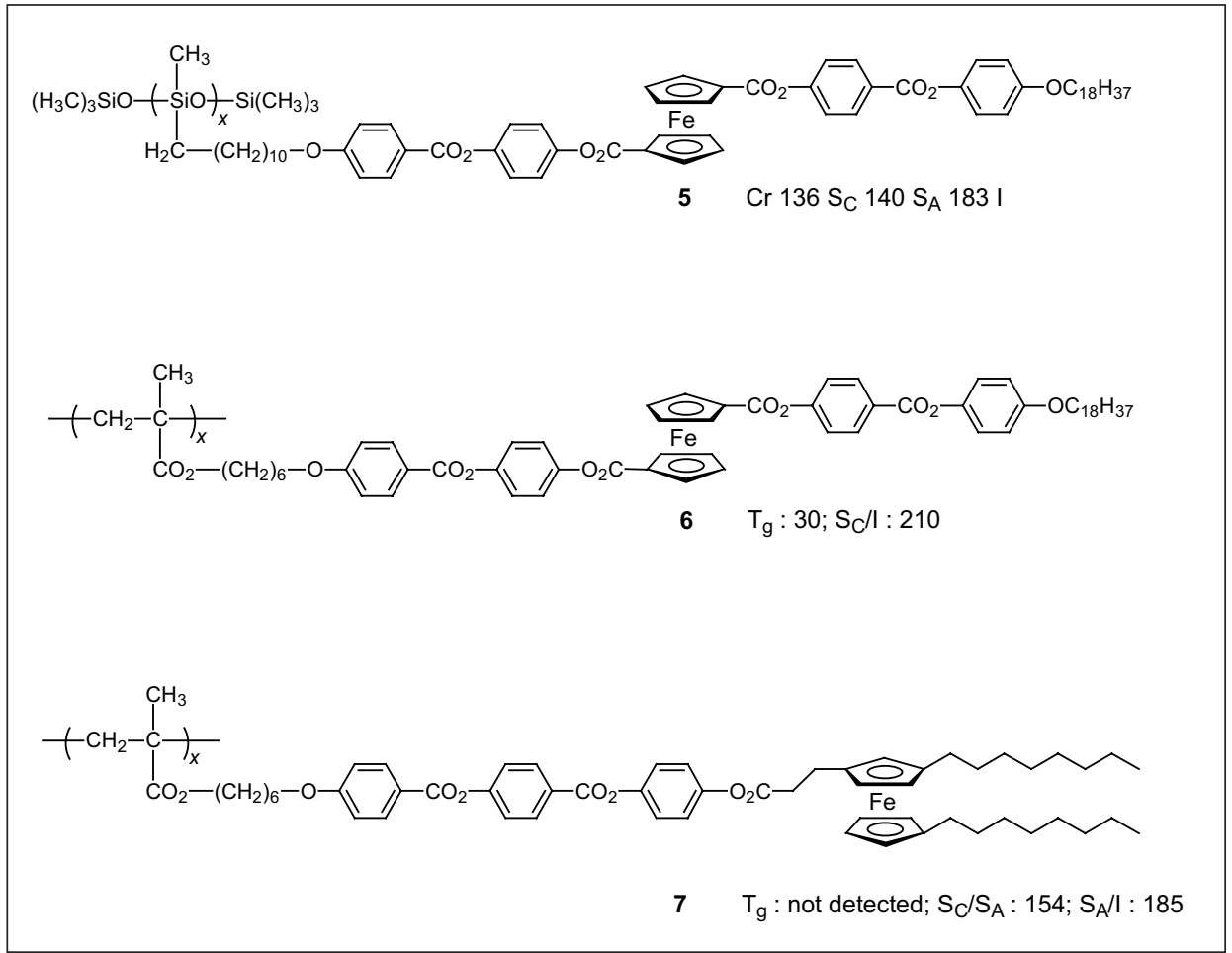

Fig. 3. Ferrocene-containing side-chain liquid-crystalline polymers and their thermal and liquidcrystalline properties. For abbreviations, see [8].

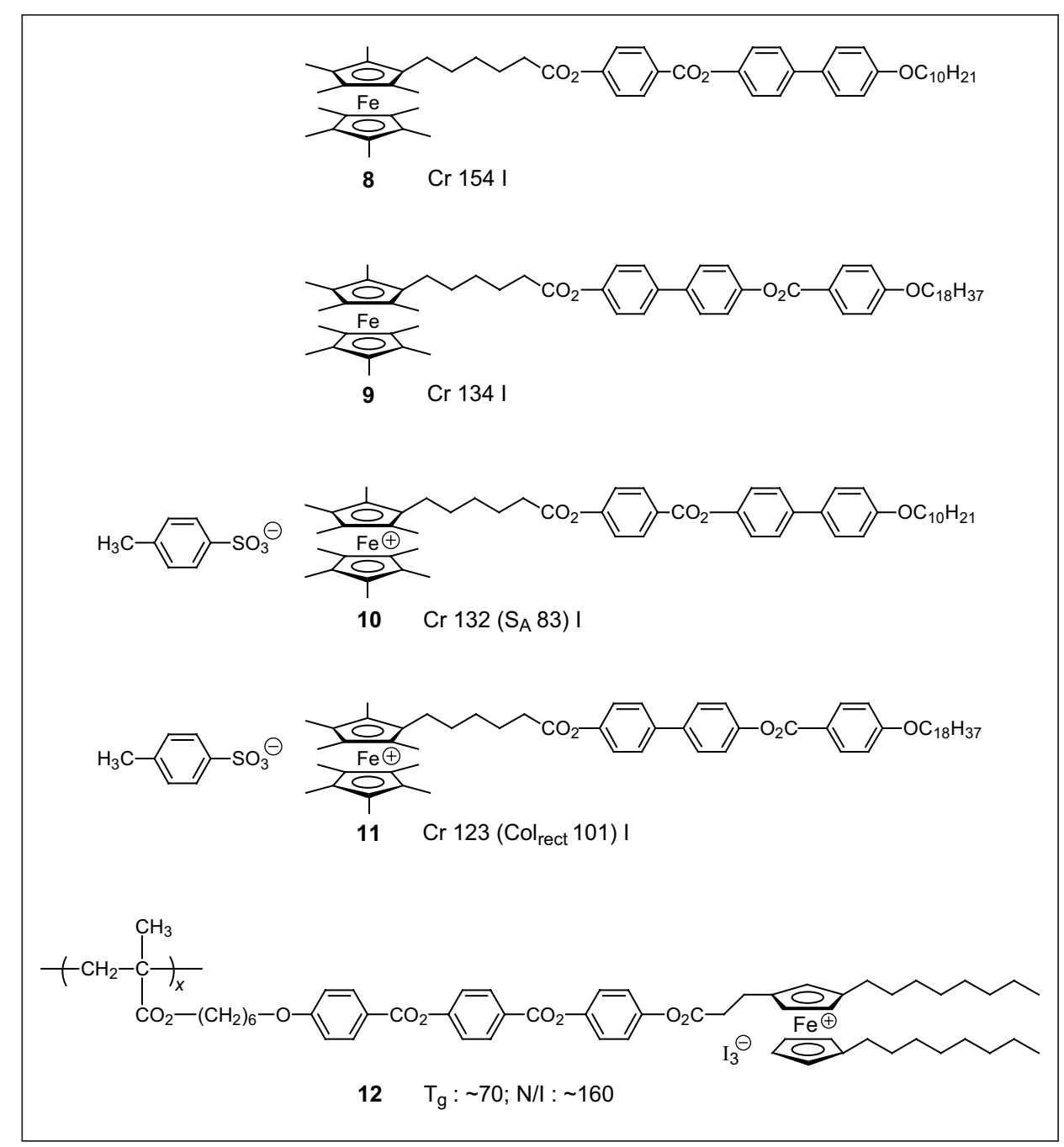

Fig. 4. Non-mesomorphic ferrocenes (8 and 9) and their corresponding liquid-crystalline ferrocenium species (10 and 11), and polyferrocenium 12 obtained from the oxidation of 7 . For abbreviations, see [8]. materials with specific functions and properties. Further developments in our group will focus on the design of mixed materials, i.e. materials containing two or more subunits, each subunit being sensitive to a specific external stimulus. Such compounds will be interesting for applications in the nanotechnologies.

\section{Acknowledgment}

R.D. acknowledges the Swiss National Science Foundation for financial support.

Received: July 14, 2003

[1] R. Deschenaux, J.W. Goodby, in 'Ferrocenes: Homogeneous Catalysis, Organic Synthesis, Materials Science', Eds. A. Togni, T. Hayashi, VCH, Weinheim, 1995, Chap. 9, p. 471-495.

[2] R. Deschenaux, J. Santiago, J. Mater. Chem. 1993, 3, 219.

[3] E. Campillos, R. Deschenaux, A.-M. Levelut, R. Ziessel, J. Chem. Soc., Dalton Trans. 1996, 2553.

[4] R. Deschenaux, B. Donnio, G. Rheinwald, F. Stauffer, G. Süss-Fink, J. Velker, J. Chem. Soc., Dalton Trans. 1997, 4351.

[5] Review on fullerene-containing liquid crystals: T. Chuard, R. Deschenaux, $J$. Mater. Chem. 2002, 12, 1944.

[6] Review on monosubstituted liquid-crystalline ferrocenes: C. Imrie, P. Engelbrecht, C. Loubser, C.W. McCleland, Appl. Organometal. Chem. 2001, 15, 1.

[7] R. Deschenaux, I. Kosztics, B. Nicolet, $J$. Mater. Chem. 1995, 5, 2291.

[8] The following abbreviations are used: $\mathrm{Cr}$ $=$ crystal, $\mathrm{Col}_{\mathrm{rec}}=$ columnar rectangular phase, $\mathrm{S}_{\mathrm{C}}=$ smectic $\mathrm{C}$ phase, $\mathrm{S}_{\mathrm{C}^{*}}=$ chiral smectic $\mathrm{C}$ phase, $\mathrm{S}_{\mathrm{A}}=$ smectic A phase, $\mathrm{N}$ $=$ nematic phase, $\mathrm{I}=$ isotropic liquid, $\mathrm{T}_{\mathrm{g}}=$ glass transition. Between parentheses $=$ monotropic phases. Temperatures are given in ${ }^{\circ} \mathrm{C}$.

[9] T. Chuard, S.J. Cowling, M. FernandezCiurleo, I. Jauslin, J.W. Goodby, R. Deschenaux, Chem. Commun. 2000, 2109.

[10] P. Singh, M.D. Rausch, R.W. Lenz, Polym Bull. 1989, 22, 247.

[11] R. Deschenaux, I. Jauslin, U. Scholten, F. Turpin, D. Guillon, B. Heinrich, Macromolecules 1998, 31, 5647.

[12] R. Deschenaux, V. Izvolenski, F. Turpin, D. Guillon, B. Heinrich, Chem. Commun. 1996, 439.

[13] R. Deschenaux, F. Turpin, D. Guillon, Macromolecules 1997, 30, 3759.

[14] J.C. Medina, I. Gay, Z. Chen, L. Echegoyen, G.W. Gokel, J. Am. Chem. Soc. 1991, 113, 365.

[15] P.D. Beer, E.L. Tite, A. Ibbotson, J. Chem Soc., Dalton Trans. 1991, 1691.

[16] A. Wiesemann, R. Zentel, G. Lieser, Acta Polym. 1995, 46, 25.

[17] A. Togni, in 'Ferrocenes: Homogeneous Catalysis, Organic Synthesis, Materials Science', Eds. A. Togni, T. Hayashi, VCH, Weinheim, 1995, Chap. 8, p. 433-469. 


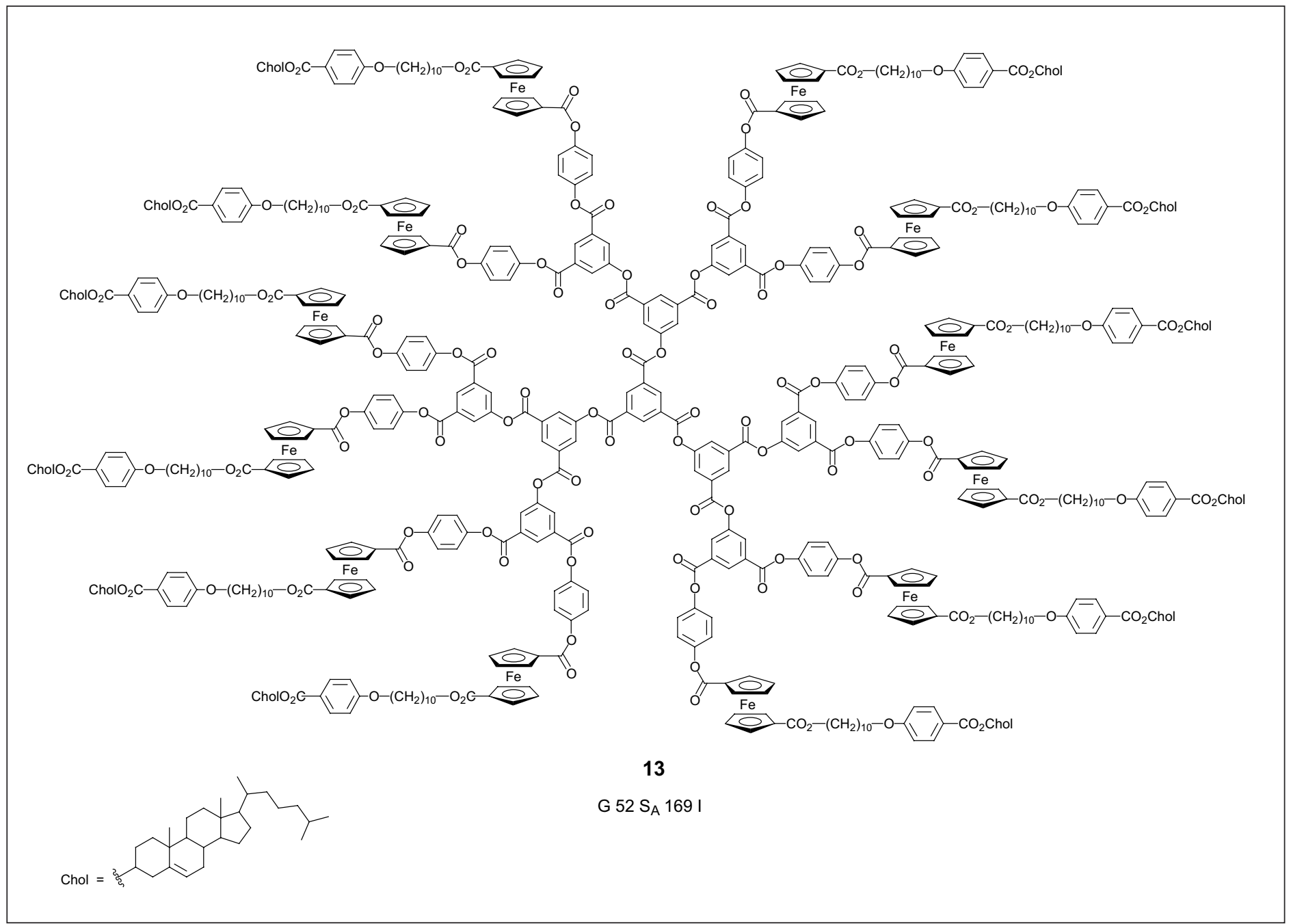

Fig. 5. Second-generation ferrocene-containing liquid-crystalline dendrimer and its thermal and liquid-crystalline properties. For abbreviations, see [8].

[18] R. Deschenaux, M. Schweissguth, A.-M. Levelut, Chem. Commun. 1996, 1275.

[19] R. Deschenaux, M. Schweissguth, M.-T. Vilches, A.-M. Levelut, D. Hautot, G.L. Long, D. Luneau, Organometallics 1999, 18, 5553.

[20] F. Turpin, D. Guillon, R. Deschenaux, Mol. Cryst. Liq. Cryst. 2001, 362, 171.

[21] 'Dendrimers and other Dendritic Polymers', Eds. J.M.J. Fréchet, D.A. Tomalia, John Wiley and Son, Chichester, 2001; 'Dendrimers and Dendrons: Concepts, Syntheses, Applications', Eds. G.R. Newkome, C.N. Moorefield, F. Vögtle, Wiley-VCH, Weinheim, Weinheim, 2001.

[22] A.W. Freeman, L.A.J. Chrisstoffels, J.M.J. Fréchet, J. Org. Chem. 2000, 65, 7612.

[23] T. Chuard, R. Deschenaux, Chimia 2001, $55,139$. 